\title{
Connectivity-Based Centroid Localization Using Distributed Dense Reference Nodes
}

\author{
Aydin Behnad, Senior Member, IEEE, \\ Xianbin Wang, Fellow, IEEE, \\ Lajos Hanzo, Fellow, IEEE, and \\ Tricia J. Willink
}

\begin{abstract}
The accuracy of centroid localization based on the connectivity information between a target node and multiple randomly scattered reference nodes is investigated. First, the accuracy of the centroid localization is analyzed in a general fading environment and it is shown that the ambiguity area of this localization technique is inversely proportional to the density of the reference nodes. Additionally, this area is at the same order and in the best case half of the ambiguity area provided by estimating the location of a target node as the location of its nearest reference node. Furthermore, increasing the transmission power of a target node will increase the accuracy of the localization, but only up to a certain limit. Then, the findings on centroid localization are verified by computer simulations.
\end{abstract}

Index Terms-Centroid localization, Poisson point process, Fifth generation mobile network (5G), Internet of Things.

\section{INTRODUCTION}

$\mathbf{T}$ HE EVER-GROWING number of networked devices in $5 \mathrm{G}[1]$ and the emergence of ubiquitous networked objects as the Internet of Things (IoT) [2] mandate a new look into the conventional approaches proposed for localization in wireless communication networks. Given the plethora of location-based civilian and industrial services as well as applications, large-scale localization techniques are becoming increasingly important, especially in indoor scenarios [3].

Thanks to the rapid increase of the network densities (e.g., from 2.2 global connections per capita in 2015 to an expected number of 3.4 in 2020 [4]) and the coexistence of different communication systems, localization techniques are evolving from infrastructure-based, passive technologies, such as the Global Positioning System (GPS), to interactive, collaborative and self-organized approaches to address the constraints of cost, complexity, and scalability.

Conventional localization processes are mainly based on the measurement of the time of arrival (TOA) [5], [6], angle of arrival (AOA) [7], [8], and/or received signal strength (RSS) [8], [9] to find the relative location of a target node with respect to a set of reference nodes (RNs) having known coordinates.

Aydin Behnad and Xianbin Wang are with the Department of Electrical and Computer Engineering, University of Western Ontario, London, ON N6A 5B9, Canada (e-mail: abehnad@uwo.ca; xianbin.wang@uwo.ca).

Lajos Hanzo is with the School of Electronics and Computer Science, University of Southampton, Southampton SO17 1BJ, U.K. (e-mail: lh@ecs.soton.ac.uk).

Tricia J. Willink is with the Defence Research and Development Canada, Ottawa, ON K1A 0Z4, Canada (e-mail: tricia.willink@drdc-rddc.gc.ca).

L. Hanzo would like to acknowledge the financial support of the ERC Advanced Fellow Grant.

(c)IEEE TVT 2018, Behnad, Wang, Hanzo, Willink
Despite their high accuracy - especially in ultra-wideband applications - the TOA and AOA are not so practical due to their high complexity and cost [10], requiring sophisticated signal processing and antenna arrays. Although the received signal strength is readily available in most types of receivers, RSS and RSS-based fingerprinting [10], [11] approaches are also highly dependent on the precise knowledge of the propagation scenario or of how accurate and frequently the RSS or the fingerprinting database is updated.

Defining localization as the process of finding the location of a target node with respect to a set of reference nodes (RNs) having determined locations, detectability of the target node by a group of RNs is the minimal information required for the success of this process. The detectability or connectivity information is typically made available in the network layer by the routing protocols, but if not, then it can be gleaned with the aid of a modest overhead in form of a single-bit connectivityflag between a target node and each RN. This allows a wireless network to support centralized collaborative large-area-based localization, which is of particular importance in IoT systems relying on low-cost low-capability devices.

The simplest method of exploiting the connectivity information to locate a target emitter is the centroid-based localization (CL), in which the centroid of the RNs detecting a target node is deemed to be its location. This method was first introduced in [12] and its accuracy was evaluated for an idealized scenario, where the RNs are placed on a grid and have the same spherical communication range. Afterwards, several weighted centroid localization schemes were introduced, in which the coordinates of each RN detecting a target node is weighted as a function of the received signal strength [13]-[16]. The CL method outperforms its variants, since it does not require any additional information other than the binary connectivity information between the target node and each of the RNs. Additionally, it is more robust in fading environments [7] and particularly suitable for indoor localization.

In this paper, we analyze the accuracy of the centroidbased localization in a Poisson field of the RNs having known locations in a general fading environment. It is shown that using centroid-based localization, the ambiguity area of a target node's location is similar to (in the best case half of) the location of its nearest RN. Additionally, we show that the localization mean squared distance error is inversely proportional to the RNs density and reduces unbounded when this density increases. However, increasing the transmission power of the target node in order to be detected by more 
RNs will no longer increase the localization accuracy, when the detection range becomes sufficiently large. We also verify the accuracy of the CL estimator numerically by computer simulations. To the best of the authors' knowledge, there is no analysis in the open literature on the accuracy of the centroid-based localization taking into account both the $R N s$ distribution and channels randomness.

The remainder of this paper is organized as follows. In Section II, the localization scenario is presented. The centroidbased localization is analyzed in Section III. Numerical results are presented in Section IV. Finally, the paper is concluded in Section V.

\section{SySTEM MODEL}

We consider a field of networked RNs, such as Wi-Fi access points, where the RN locations constitute a homogeneous Poisson point process (PPP) with density $\lambda$. As shown in Fig. 1, a target node $\mathrm{T}$ at $\left(x_{0}, y_{0}\right)$ is detected by (or is connected to) each RN provided that the RN lies within its connectivity range $R$. The range $R$ is a random variable and depends on several parameters, including the transmission power and the channel's fading gain. If the distance between $\mathrm{T}$ and an $\mathrm{RN}$ is $r, \mathrm{~T}$ is detected by that $\mathrm{RN}$ with the probability of

$$
\Phi(r)=\operatorname{Pr}\{R>r\} .
$$

We also assume that the connectivity range in any direction around $\mathrm{T}$ is independent of the other directions and that the function $\Phi(\cdot)$ is determined. Additionally, we assume that $\mathbb{E}\left[R^{4}\right]$ (and consequently $\mathbb{E}\left[R^{2}\right]$ ) are finite, where $\mathbb{E}[\cdot]$ denotes the expectation operator.

The locations of the RNs detecting the target node $T$ is indexed as $\left\{\left(X_{k}, Y_{k}\right)\right\}_{k=1}^{N}$, where the indices $k$ for the abscissa $X_{k}$ and ordinate $Y_{k}$ are not the proximity indices, since we do not know which RN is closer to $\mathrm{T}$ and which one is farther away from $\mathrm{T}$. In fact, due to the fading effects, $\left\{\left(X_{k}, Y_{k}\right)\right\}_{k=1}^{N}$ are not necessarily the locations of the $N$ nearest RNs to T and the node indexing can be arbitrary.

Furthermore, it should be noted that although we assume that the RNs are distributed over the entire plane, our analysis also holds, when they are distributed uniformly over a large region with density $\lambda$, where the target node and the $N$ detecting RNs are located sufficiently far from the region boundaries.

In the next section we analyze the accuracy of the centroidbased localization scheme in a generalized fading environment of the above-mentioned system model, which is the main problem considered in this paper.

\section{Centroid-Based Localization}

Perhaps the simplest unbiased location estimator of the target node $\mathrm{T}$ is constituted by the arithmetic mean of the $\mathrm{RN}$ locations detecting $\mathrm{T}$, which is given by

$$
(\hat{X}, \hat{Y})=\left(\frac{1}{N} \sum_{k=1}^{N} X_{k}, \frac{1}{N} \sum_{k=1}^{N} Y_{k}\right) \text {. }
$$

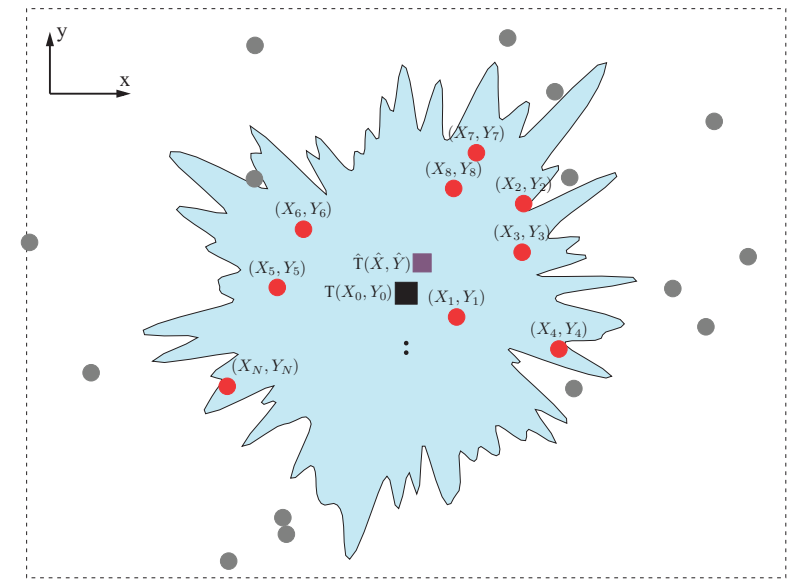

Fig. 1. The target node $\mathrm{T}$ in a Poisson field of reference nodes is connected to (detected by) the nodes in the target connectivity (detectability) region (blue region). The reference nodes connecting to $\mathrm{T}$ are indexed in an arbitrary fashion.

Since $(\hat{X}, \hat{Y})$ is the centroid of points $\left\{\left(X_{k}, Y_{k}\right)\right\}_{k=1}^{N}{ }^{1}$, this localization scheme is the centroid-based localization, hence we simply refer to it as the centroid estimator.

For the centroid localization, the distance error between the estimated location of the target node $\mathrm{T}$ and its exact location is obtained as

$$
D_{\mathrm{e}}=\sqrt{\left(\hat{X}-x_{0}\right)^{2}+\left(\hat{Y}-y_{0}\right)^{2}} .
$$

Given $D_{\mathrm{e}}=d$, the exact location of $\mathrm{T}$ is within a circular region of radius $d$, centered at $(\hat{X}, \hat{Y})$. Hence, we define the effective ambiguity area as

$$
A_{\mathrm{e}}=\mathbb{E}\left[\pi D_{\mathrm{e}}^{2}\right]=\pi \mathbb{E}\left[D_{\mathrm{e}}^{2}\right] .
$$

The following theorem provides measures for the accuracy of the centroid localization method.

Theorem 1: The centroid localization given by (2) is unbiased. Additionally, the mean squared distance error (MSDE) and the effective ambiguity area for the location of the target node $\mathrm{T}$ are given by

$$
\mathbb{E}\left[D_{\mathrm{e}}^{2}\right]=\mathrm{G}\left(\lambda \pi \overline{R^{2}}\right) \frac{\overline{R^{4}}}{2 \overline{R^{2}}}
$$

and

$$
A_{\mathrm{e}}=\mathrm{G}\left(\lambda \pi \overline{R^{2}}\right) \frac{\pi \overline{R^{4}}}{2 \overline{R^{2}}}
$$

respectively, where we have

$$
\overline{R^{i}} \triangleq \mathbb{E}\left[R^{i}\right]=\int_{0}^{\infty} i r^{i-1} \Phi(r) \mathrm{d} r, \quad i=2,4
$$

and

$$
\begin{aligned}
\mathrm{G}(z) & \triangleq \frac{1}{e^{z}-1} \int_{0}^{z} \frac{e^{x}-1}{x} \mathrm{~d} x \\
& =\frac{\operatorname{Shi}(z)+\operatorname{Chi}(z)-\ln (z)-\gamma}{e^{z}-1}
\end{aligned}
$$

\footnotetext{
${ }^{1}$ Note that the localization accuracy is meaningful when the target node is detected by at least one RN. Hence, our analysis is conditioned on $N \geqslant 1$, as $N=0$ means that no target node has been detected and the centroid estimator in (2) is undefined.
} 
for $z>0$, in which $\operatorname{Shi}(\cdot), \operatorname{Chi}(\cdot)$, and $\gamma=0.57721$ are the hyperbolic sine integral function [17, eq. 5.2.3], hyperbolic cosine integral function [17, eq. 5.2.4], and Euler's constant $[17$, p. 3], respectively.

\section{Proof: See Appendix.}

The argument of function $\mathrm{G}(\cdot)$ in (5) and (6), i.e.,

$$
\lambda_{N}=\lambda \pi \overline{R^{2}}
$$

represents the average number of RNs detecting T. To analyze the performance of the centroid estimator, we first have to characterize the behavior of the function $\mathrm{G}(z)$ in terms of $z$. It can be shown that $\lim _{z \rightarrow 0^{+}} \mathrm{G}(z)=1, \lim _{z \rightarrow \infty} z \mathrm{G}(z)=1$, and $\mathrm{G}^{\prime}(z)<0$ for $z>0$. Therefore $\mathrm{G}(z)$ has the following properties:

1) $\mathrm{G}\left(0^{+}\right)=1$;

2) $\mathrm{G}(z) \cong \frac{1}{z}$ for $z \gg 1$;

3) $\mathrm{G}(z)$ is a monotonically decreasing function of $z>0$.

Here, we consider two extreme cases for the average number of nodes detecting $\mathrm{T}$, namely, very small values and very large values of $\lambda_{N}$.

\section{A. Very small values of $\lambda_{N}$}

When the density of RNs is low or the connectivity range is statistically speaking small so that $\lambda_{N} \ll 1$, taking the expectation over the cases where at least one RN detects $\mathrm{T}$, the MSDE and the effective ambiguity area are, respectively, reduced to

$$
\mathbb{E}\left[D_{\mathrm{e}}^{2}\right]=\frac{\overline{R^{4}}}{2 \overline{R^{2}}} \quad, \quad A_{\mathrm{e}}=\frac{\pi \overline{R^{4}}}{2 \overline{R^{2}}} \quad\left(\lambda \pi \overline{R^{2}} \ll 1\right) .
$$

In this case, $\mathrm{T}$ is only detected by its nearest neighbor with a probability close to 1 and the centroid estimate of the location of $\mathrm{T}$ is the location of its nearest neighbor. To verify this fact, we assume $R=R_{\mathrm{NN}}$, where $R_{\mathrm{NN}}$ is the distance to the nearest neighbor of T. Thus, we expect $D_{\mathrm{e}}=R_{\mathrm{NN}}$ and using (10), we have:

$$
\mathbb{E}\left[R_{\mathrm{NN}}^{2}\right]=\frac{\mathbb{E}\left[R_{\mathrm{NN}}^{4}\right]}{2 \mathbb{E}\left[R_{\mathrm{NN}}^{2}\right]}
$$

It may be readily shown (using [18, eq. (10)]) that this is the case for the distance measured for the nearest neighbor in a homogeneous two-dimensional PPP.

\section{B. Very large values of $\lambda_{N}$}

When $\lambda_{N}$ is sufficiently large so that the approximation $\mathrm{G}(z) \cong \frac{1}{z}$ becomes accurate enough, from (5) and (6) we obtain

$$
\mathbb{E}\left[D_{\mathrm{e}}^{2}\right]=\frac{1}{2 \pi \lambda} \frac{\overline{R^{4}}}{\left(\overline{R^{2}}\right)^{2}} \quad, \quad A_{\mathrm{e}}=\frac{1}{2 \lambda} \frac{\overline{R^{4}}}{\left(\overline{R^{2}}\right)^{2}} \quad\left(\lambda \pi \overline{R^{2}} \gg 1\right) .
$$

Using numerical values of $\mathrm{G}(\cdot)$, the approximation errors involved in using the simplified expressions (12) are less than $10 \%, 5 \%$, and $1 \%$, when $\lambda_{N}$ is higher than $12.26,22.12$, and 102.02 , respectively.
An immediate result of (12) is that if the connectivity range is scaled as $c R$ for a constant $c>0$, which is achieved by increasing the transmit power of $\mathrm{T}$, the localization accuracy will not change. Note that this holds as long as $\lambda_{N}$ is sufficiently large. On the other hand, $\lambda_{N}$ becomes arbitrarily large when $c$ is sufficiently high (see (9)). Therefore, increasing the transmit power of $\mathrm{T}$ beyond a certain threshold so that $\mathrm{T}$ is detected by more RNs will no longer improve the accuracy of the centroid localization. For instance, when $\lambda \pi \overline{R^{2}} \geqslant 12.26$, increasing the transmission power will no longer reduce the effective ambiguity region by more than $10 \%$. However, increasing the RNs density $\lambda$ will monotonically and unboundedly improve the accuracy of the localization.

Using [18, eq. (10)], the average squared distance to the nearest-neighbor $\mathrm{RN}$ is given by $\mathbb{E}\left[R_{\mathrm{NN}}^{2}\right]=1 /(\pi \lambda)$. Additionally, since $\operatorname{var}\left(R^{2}\right)=\overline{R^{4}}-\left(\overline{R^{2}}\right)^{2} \geqslant 0$, we have $\overline{R^{4}} \geqslant\left(\overline{R^{2}}\right)^{2}$ and the equality holds when $R$ is constant, i.e., in the absence of fading. Hence, the MSDE and the effective ambiguity area in (12) satisfy

$$
\mathbb{E}\left[D_{\mathrm{e}}^{2}\right] \geqslant \frac{1}{2} \mathbb{E}\left[R_{\mathrm{NN}}^{2}\right], \quad A_{\mathrm{e}} \geqslant \frac{1}{2} \mathbb{E}\left[\pi R_{\mathrm{NN}}^{2}\right] \quad\left(\lambda \pi \overline{R^{2}} \gg 1\right) .
$$

As a result, in a dense network, the effective ambiguity area of the centroid localization is at best half of the effective ambiguity area provided by the nearest neighbor localization, where the location of the nearest neighbor $\mathrm{RN}$ is considered to be the target location. However, note that since the location of the target node is not known, its nearest neighbor RN cannot be determined from the information constituted by its connectivity to the RNs. Conversely, based on our discussion, the centroid-based localization can be used for determining, which RN the target is likely to be close to.

As an example, assume that the centroid-based localization is used in a Rayleigh fading environment so that the power received by an RN at distance $d$ from $\mathrm{T}$ is given by

$$
P_{\mathrm{r}}=P_{\mathrm{th}}\left(\frac{d_{0}}{d}\right)^{\alpha} \Omega
$$

where $P_{\text {th }}$ is the minimum received power required for reliable communication, $d_{0}$ is the maximum communication range in the absence of fading (i.e., $\Omega=1$ ), $\alpha$ is the path-loss exponent, usually obeying $2 \leqslant \alpha \leqslant 7$, and $\Omega$ is a unit-mean exponential random variable. From (14) associated with $P_{\mathrm{r}}=P_{\text {th }}$, the maximum communication range is also a random variable obeying

$$
d_{\max }=d_{0} \Omega^{\frac{1}{\alpha}} .
$$

Hence, the connectivity function $\Phi(\cdot)$ is given by

$$
\Phi(r)=\operatorname{Pr}\left\{d_{\max }>r\right\}=\operatorname{Pr}\left\{\Omega>\left(\frac{r}{d_{0}}\right)^{\alpha}\right\}=e^{-\left(\frac{r}{d_{0}}\right)^{\alpha}}
$$

By substituting $\Phi(r)$ from (16) into (7) and using [19, eq. 2.3.18.2 \& Appendix II.1], we arrive at:

$$
\overline{R^{4}}=\frac{4}{\alpha} \Gamma\left(\frac{4}{\alpha}\right) d_{0}^{4} \quad, \quad \overline{R^{2}}=\frac{2}{\alpha} \Gamma\left(\frac{2}{\alpha}\right) d_{0}^{2}
$$

and, therefore, eqs. (5) and (12) are reduced to

$$
\mathbb{E}\left[D_{\mathrm{e}}^{2}\right]=\mathrm{G}\left(2 \lambda \pi d_{0}^{2} \Gamma(2 / \alpha) / \alpha\right) \frac{\Gamma(4 / \alpha)}{\Gamma(2 / \alpha)} d_{0}^{2}
$$




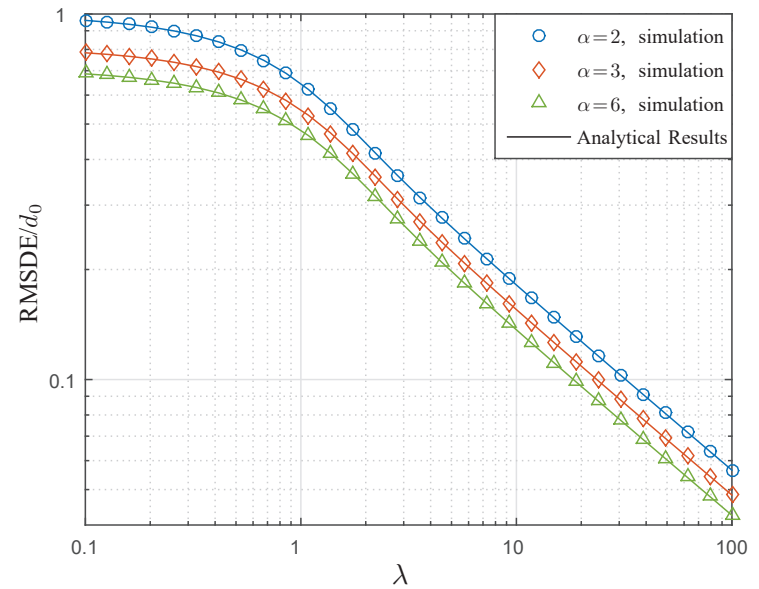

Fig. 2. RMSDE, normalized to $d_{0}$, in terms of the density $\lambda$, for different values of the paht-loss exponent $\alpha$ and in a Rayleigh fading environment. The analytical results were computed from Eq. (18).

and

$$
\mathbb{E}\left[D_{\mathrm{e}}^{2}\right]=\frac{\alpha}{2 \lambda \pi \mathcal{B}(2 / \alpha, 2 / \alpha)}, \quad \lambda_{N} \gg 1
$$

using [17, eq. 6.2.2], where $\mathcal{B}(\cdot, \cdot)$ is the beta function [17, eq. 6.2.1].

\section{Numerical Results AND Simulation}

In this section, we evaluate the performance of the centroidbased localization and verify the accuracy of the analytical results obtained. Our Monte Carlo simulation results in this section are based on $10^{6}$ independent realizations of the system.

Fig. 2 shows the root mean square distance error (RMSDE), normalized to $d_{0}=1$, of localizing a target node in a network having the density $\lambda$, for different values of the path-loss exponent $\alpha$. The simulation results of this figure are for a Rayleigh fading environment, while the analytical results are based on equation (18). As observed, the simulation results confirm the accuracy of the analytical results. Furthermore, when $\lambda$ becomes small, the normalized RMSDEs become almost constant. Using (10) with (17), we find the normalized RMSDEs for small values of the RN densities as 1.00, 0.85, and 0.75 for $\alpha=2,3$, and 6 , respectively, which are consistent with the results shown in this figure. On the other side of the curves, when the network density becomes sufficiently high, the normalized RMSDEs decrease linearly in a log-log plot upon increasing the network's density, which is expected from (19). In other words, in a dense network, the RMSDE is proportional to the network's density as $1 / \sqrt{\lambda}$. Moreover, the normalized RMSDE attained for higher values of the path-loss exponent is reduced. of it. This is because when $\alpha$ increases, the maximum communication range fluctuations in different directions are statistically reduced, as seen in (15). Hence, for a fixed density, the variation of $\mathrm{T}$ to RNs distances is decreased, which leads to reduced localization errors.

In Fig. 3, the RMSDE for the centroid estimator to localize a target node having Rayleigh fading channels with respect to the RNs is shown versus the increased transmission power. The results are for RN density $\lambda=1$, and $d_{0}=1$ when the power

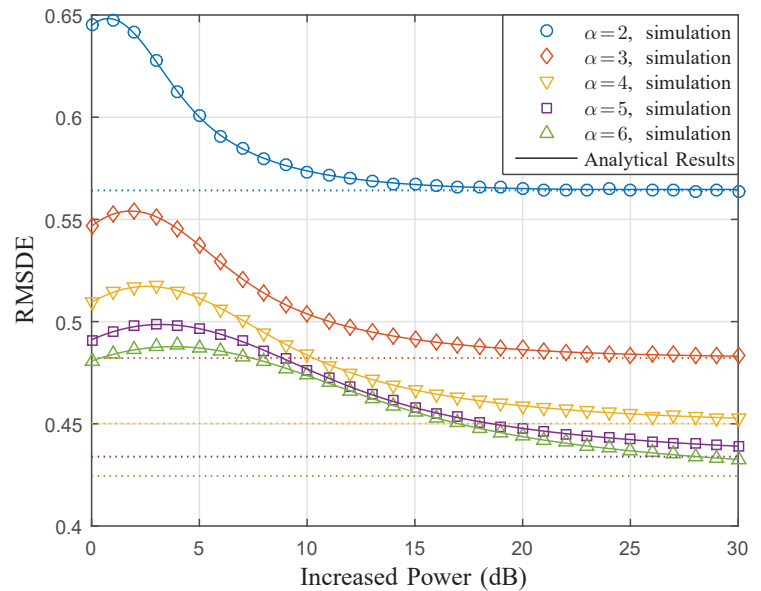

Fig. 3. The RMSDE of the centroid estimator in terms of the increased power for different values of the path-loss exponent $\alpha$ and in a Rayleigh fading environment $\left(\lambda=1\right.$, and $d_{0}=1$ for $0 \mathrm{~dB}$ increased power). The analytical result was computed from Eq. (18). Dotted lines are the asymptotic values computed from Eq. (19).

increase is $0 \mathrm{~dB}$. Hence, using (14), when the transmission power increases $p \mathrm{~dB}, d_{0}$ increases to $10^{\frac{p}{10 \alpha}} d_{0}$. As shown in this figure, the localization error slightly increases, when the transmit power is increased from low values. This is due to the fact that when the transmit power is low, it is more likely that the target node is not detected by any of the network nodes. However, if they are detected by chance, the detecting node(s) are likely to be very close to the target node and the detection RMSDE would be low. When the transmission power increases, the detection probability increases, but the detecting nodes will not necessarily be close to the target node. Additionally, the normalized RMSDE becomes asymptotically constant, when the transmission power value is sufficiently increased, as also observed in (19). The dotted lines show the asymptotic values obtained from (19). Note that the analytical results obtained from (18) for different values of the path-loss exponent are corroborated by the simulation results.

In the simulations given by Figs. 2 and 3, it was assumed that the target is detected by at least one RN. Additionally, the RNs are randomly scattered as a PPP. Here, we perform the same simulations but with including the effect of the cases where the target is not detected or the RNs have a regular placement. The results for $\alpha=4$ are given by Figs. 4 and 5, respectively. In the new simulations, we assumed that the target node has a symmetrical distribution in a wide area around a center point, with a root mean square distance to that center point of RMSD $=5$. Whenever the target is not detected, its location is estimated as the center point. We also considered the square grid placement of the RNs in our simulations, where the center point is a randomly selected point with respect to the square grid.

As observed in Figs. 4 and 5, when the RN density or the target transmission power become sufficiently high, the RMSDE for all cases and the target-detected cases are almost the same. This is indeed expected, because increasing either of these two parameters results in increasing the probability of target detection. Furthermore, Fig. 4 indicates that, similar 


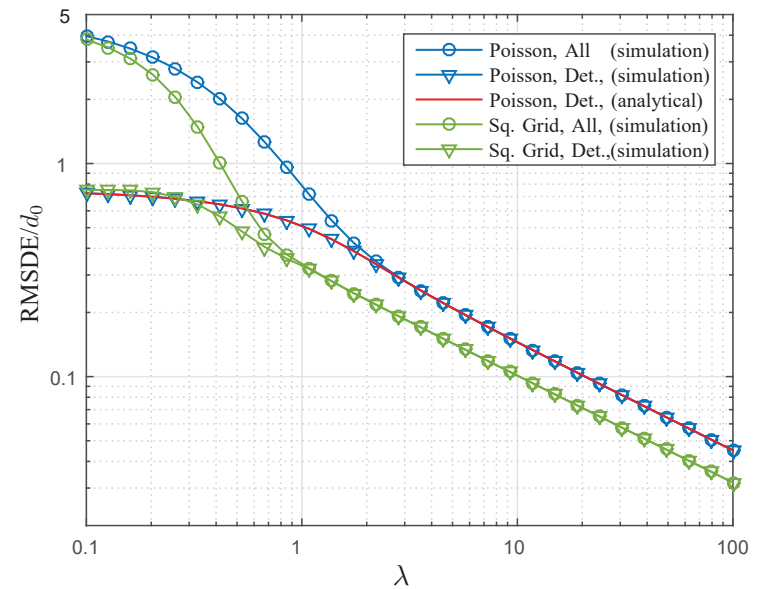

Fig. 4. RMSDE, normalized to $d_{0}$, in terms of the density $\lambda$, among all simulated (All) and target-detected (Det.) cases. The reference nodes have Poisson distribution and square-grid placement in a Rayleigh fading environment with $\alpha=4$.

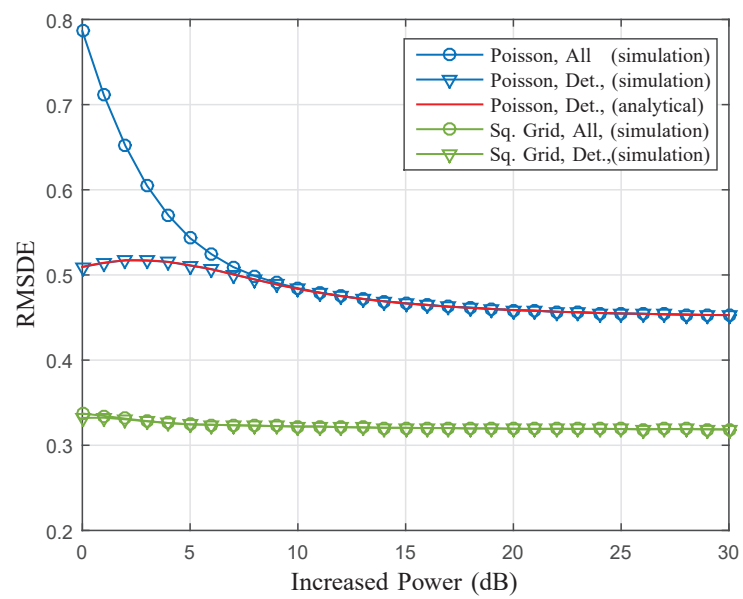

Fig. 5. The RMSDE of the centroid estimator in terms of the increased power among all simulated (All) and target-detected (Det.) cases, for $\alpha=4$ and in a Rayleigh fading environment $\left(\lambda=1\right.$, and $d_{0}=1$ for $0 \mathrm{~dB}$ increased power $)$

to the PPP case, the RMSDE for the square-grid placement case decreases asymptotically as $1 / \sqrt{\lambda}$ for large $\lambda$, but it has a better accuracy at the expense of the regular RNs placement. When $\lambda$ is very small, both distributions of the RNs have the same performance, since the target node either is not detected, or it is detected by one RN and hence it makes no difference what the distribution of the RNs is. Moreover, Fig. 5 shows that similarly to the PPP case, the localization error becomes asymptotically constant in the square-grid placement of RNs, when the target transmission power becomes sufficiently high, but this constant RMSDE is lower than that of the PPP scenario.

\section{CONCLUSiOnS}

We have evaluated the performance of the connectivitybased localization in which the only information used for the localization process is whether a target node is connected to each RN or not. It has been shown that in a dense network, if a target node's position is estimated by the centroid of RNs detecting that target node, the squared distance error of the localization is inversely proportional to the density of the RNs. Furthermore, this error is improved by increasing the transmit power up to a certain limit. Moreover, the ambiguity area of this localization method is on the same order and in its best case half of the ambiguity area provided by mapping the location of the target node at the location of its nearest RN.

This work can be extended in several directions. For example, the performance of the centroid localization can be analyzed for other path-loss models and other distributions of the RNs. Our techniques can also be invoked both in indoor and outdoor experimental tests. Another interesting extension is to analyze the performance of the weighted centroid localization schemes within the same system model considered in this paper.

\section{APPENDIX}

Without losing generality, we assume that $\mathrm{T}$ is at the origin, i.e., $\left(x_{0}, y_{0}\right)=(0,0)$. By thinning [20, Def. 3.6] the homogeneous PPP of the location of all RNs to the PPP of the location of RNs detecting $\mathrm{T}$, we obtain the intensity function of the latter PPP as $\lambda_{2 \mathrm{D}}(r)=\lambda \Phi(r)$ [20, Prep. 3.7] in polar $(r, \theta)$ coordinates. Therefore, the total number $N$ of RNs detecting $\mathrm{T}$ is a Poisson random variable with a mean of

$$
\lambda_{N}=\int_{0}^{2 \pi} \int_{0}^{\infty} \lambda_{2 \mathrm{D}}(r) r \mathrm{~d} r \mathrm{~d} \theta=2 \pi \lambda \int_{0}^{\infty} r \Phi(r) \mathrm{d} r .
$$

From the definition of $\Phi(\cdot)$ in (1), we find that $\Phi(\cdot)$ is the complementary cumulative distribution function of $R$. Therefore, using [21, Sec. 1.10.2-(d)], the equalities in (7) hold and we can write $\lambda_{N}$ in (20) as given by (9).

The abscissas of the RNs detecting T, i.e., $\left\{X_{n}\right\}_{n=1}^{N}$ constitute a one-dimensional PPP with density

$$
\lambda_{\mathrm{x}}(x)=\int_{-\infty}^{\infty} \lambda_{2 \mathrm{D}}\left(\sqrt{x^{2}+y^{2}}\right) d y=2 \lambda \int_{0}^{\infty} \Phi\left(\sqrt{x^{2}+y^{2}}\right) d y .
$$

Since the integrand in (21) is symmetric with respect to $x$, using the change of variable $y=|x| \tan (\theta)$, we obtain

$$
\lambda_{\mathrm{x}}(x)=2 \lambda \int_{0}^{\frac{\pi}{2}} \Phi\left(\frac{|x|}{\cos (\theta)}\right) \frac{|x|}{\cos ^{2}(\theta)} \mathrm{d} \theta .
$$

Conditioned on $N=n$, the distribution of each $\mathrm{RN}$, independent of the other RNs, has the following distribution [20, Def. 3.2-(ii)]

$$
\mathrm{f}_{X_{k}}(x)=\frac{\lambda_{\mathrm{x}}(x)}{\int_{-\infty}^{\infty} \lambda_{\mathrm{x}}(x) \mathrm{d} x}
$$

for $k=1,2, \ldots, n$. The denominator of (23) gives the average number of RNs detecting T, i.e., $\lambda_{N}$. Consequently, using (9), we have

$$
\mathrm{f}_{X_{k}}(x)=\frac{2}{\pi \overline{R^{2}}} \int_{0}^{\frac{\pi}{2}} \Phi\left(\frac{|x|}{\cos (\theta)}\right) \frac{|x|}{\cos ^{2}(\theta)} \mathrm{d} \theta .
$$

The second moment of $X_{k}$ is derived from (24) as

$$
\begin{aligned}
\mathbb{E}\left[X_{k}^{2}\right] & =\int_{-\infty}^{\infty} x^{2} \mathrm{f}_{X_{k}}(x) \mathrm{d} x \\
& =\frac{4}{\pi \overline{R^{2}}} \int_{0}^{\frac{\pi}{2}} \int_{0}^{\infty} \Phi\left(\frac{x}{\cos (\theta)}\right) \frac{x^{3}}{\cos ^{2}(\theta)} \mathrm{d} x \mathrm{~d} \theta .
\end{aligned}
$$


Using the change of variable $r=x / \cos (\theta)$, we arrive at:

$$
\mathbb{E}\left[X_{k}^{2}\right]=\frac{1}{\pi \overline{R^{2}}} \int_{0}^{\frac{\pi}{2}} \cos ^{2}(\theta) \mathrm{d} \theta \int_{0}^{\infty} 4 r^{3} \Phi(r) \mathrm{d} r=\frac{\overline{R^{4}}}{4 \overline{R^{2}}},
$$

where in the last equality we used (7). Since $\mathbb{E}\left[X_{k}^{2}\right]$ is finite, $\mathbb{E}\left[X_{k}\right]$ is also finite and due to the fact that $f_{X_{k}}(x)$ is a symmetric function with respect to $x$, we get

$$
\mathbb{E}\left[X_{k}\right]=0 .
$$

A direct result of (27) is that $\hat{X}$ is an unbiased estimator for the abscissa of T. The mean squared error (MSE) of this estimator is obtained as follows

$$
\begin{aligned}
& \mathbb{E}\left[\hat{X}^{2}\right]=\mathbb{E}\left[\left(\frac{1}{N} \sum_{k=1}^{N} X_{k}\right)^{2} \mid N \geqslant 1\right] \\
& =\mathbb{E}_{N}\left[\frac{1}{N^{2}} \sum_{k=1}^{N} \sum_{j=1}^{N} \mathbb{E}\left[X_{k} X_{j} \mid N=n \geqslant 1\right] \mid N \geqslant 1\right] .
\end{aligned}
$$

From the properties of PPPs, given $N=n \geqslant 1$, random variables $X_{i}$ and $X_{j}$ are independent when $i \neq j$. Thus, using (27), the summand term in (28) is equal to zero if $i \neq j$ and is given by (26) when $i=j$. Hence, (28) is reduced to

$\mathbb{E}\left[\hat{X}^{2}\right]=\mathbb{E}_{N}\left[\frac{1}{N^{2}} \times N \frac{\overline{R^{4}}}{4 \overline{R^{2}}} \mid N \geqslant 1\right]=\frac{\overline{R^{4}}}{4 \overline{R^{2}}} \mathbb{E}\left[\frac{1}{N} \mid N \geqslant 1\right]$.

Since $N$ is a Poisson random variable with mean $\lambda_{N}$, the probability mass function of $N$, given $N \geqslant 1$, is derived as

$$
\mathrm{P}_{N}(n \mid N \geqslant 1)=\frac{1}{1-e^{-\lambda_{N}}} e^{-\lambda_{N}} \frac{\lambda_{N}^{n}}{n !}, \quad n=1,2, \ldots .
$$

Therefore, the expectation term in (29) is obtained as

$$
\begin{aligned}
\mathbb{E}\left[\frac{1}{N} \mid N \geqslant 1\right] & =\sum_{n=1}^{\infty} \frac{1}{n} \mathrm{P}_{N}(n \mid N \geqslant 1)=\frac{1}{e^{\lambda_{N}}-1} \sum_{n=1}^{\infty} \frac{\lambda_{N}^{n}}{n ! n} \\
& =\frac{1}{e^{\lambda_{N}}-1} \int_{0}^{\lambda_{N}} \frac{e^{x}-1}{x} \mathrm{~d} x
\end{aligned}
$$

where we have used [19, eq. 5.2.8.3] in the last equality. Hence, (29) is reduced to

$$
\mathbb{E}\left[\hat{X}^{2}\right]=\mathrm{G}\left(\lambda_{N}\right) \frac{\overline{R^{4}}}{4 \overline{R^{2}}}=\mathrm{G}\left(\lambda \pi \overline{R^{2}}\right) \frac{\overline{R^{4}}}{4 \overline{R^{2}}},
$$

where $\mathrm{G}(z)$ is given by (8). Function $\mathrm{G}(\cdot)$ can also be written in terms of $\operatorname{Shi}(\cdot)$ and $\operatorname{Chi}(\cdot)$ as given by $(8 b)$, using [17, eqs. 5.2.3 and 5.2.4].

Using the same approach, it can be shown that $\hat{Y}$ is an unbiased estimator for the ordinate of the location of $\mathrm{T}$ and $\mathbb{E}\left[\hat{Y}^{2}\right]$ is obtained equal to $\mathbb{E}\left[\hat{X}^{2}\right]$ given in (26). Therefore,

$$
\mathbb{E}\left[D_{\mathrm{e}}^{2}\right]=\mathbb{E}\left[\hat{X}^{2}+\hat{Y}^{2}\right]=2 \mathbb{E}\left[\hat{X}^{2}\right]
$$

and (5) and then (6) are obtained by substituting (32) into (33), and the proof is complete.

\section{REFERENCES}

[1] J. G. Andrews, S. Buzzi, W. Choi, S. V. Hanly, A. Lozano, A. C. K. Soong, and J. C. Zhang, "What will 5G be?" IEEE J. Sel. Areas Commun., vol. 32, no. 6, pp. 1065-1082, Jun. 2014.

[2] A. Al-Fuqaha, M. Guizani, M. Mohammadi, M. Aledhari, and M. Ayyash, "Internet of things: A survey on enabling technologies, protocols, and applications," IEEE Commun. Surveys Tuts., vol. 17, no. 4, pp. 2347-2376, Fourth Quarter 2015.

[3] S. Feng, X. Li, R. Zhang, M. Jiang, and L. Hanzo, "Hybrid positioning aided amorphous-cell assisted user-centric visible light downlink techniques," IEEE Access, vol. 4, pp. 2705 -2713, May 2016.

[4] Cisco, "2016 Cisco VNI Complete Forecast," white paper at Cisco.com.

[5] T.-K. Le and N. Ono, "Closed-form and near closed-form solutions for TDOA-based joint source and sensor localization," IEEE Trans. Signal Process., vol. 65, no. 5, pp. 1207-1221, Mar. 2017.

[6] G. Wang, H. Chen, Y. Li, and N. Ansari, "NLOS error mitigation for TOA-based localization via convex relaxation," IEEE Trans. Wireless Commun., vol. 13, no. 8, pp. 4119 - 4131, Aug. 2014.

[7] N. Garcia, H. Wymeersch, E. G. Larsson, A. M. Haimovich, and M. Coulon, "Direct localization for massive MIMO," IEEE Trans. Signal Process., vol. 65, no. 10, pp. 2475-2487, May 2017.

[8] S. Tomic, M. Beko, and R. Dinis, "3-D Target localization in wireless sensor networks using RSS and AoA measurements," IEEE Trans. Veh. Technol., vol. 66, no. 4, pp. 3197 - 3210, Apr. 2015.

[9] _ - "RSS-Based localization in wireless sensor networks using convex relaxation: Noncooperative and cooperative schemes," IEEE Trans. Veh. Technol., vol. 64, no. 5, pp. 2037 - 2050, May 2015.

[10] K. Lin, M. Chen, J. Deng, M. M. Hassan, and G. Fortino, "Enhanced fingerprinting and trajectory prediction for IoT localization in smart buildings," IEEE Trans. Autom. Sci. Eng., vol. 13, no. 3, pp. 1294-1307, Jul. 2016.

[11] X. Wang, L. Gao, S. Mao, and S. Pandey, "CSI-based fingerprinting for indoor localization: A deep learning approach," IEEE Trans. Veh. Technol., vol. 66, no. 1, pp. 763-776, Jan. 2017.

[12] N. Bulusu, J. Heidemann, and D. Estrin, "GPS-less low-cost outdoor localization for very small devices," IEEE Personal Commun. Mag., vol. 7, no. 5, pp. 28-34, Oct. 2000.

[13] J. Blumenthal, R. Grossmann, F. Golatowski, and D. Timmermann, "Weighted centroid localization in Zigbee-based sensor networks," in Proc. IEEE Int. Symp. Intell. Signal Process, Oct. 2007, pp. 1-6.

[14] C. Laurendeau and M. Barbeau, "Centroid localization of uncooperative nodes in wireless networks using a relative span weighting method," EURASIP J. Wireless Commun. Netw., vol. 2010, Article ID 567040, 2010.

[15] J. Wang, P. Urriza, Y. Han, and D. Cabric, "Weighted centroid localization algorithm: Theoretical analysis and distributed implementation," IEEE Trans. Wireless Commun., vol. 10, no. 10, pp. 3403-3413, Oct. 2011.

[16] S. Chaudhari and D. C. Senior, "Cyclic weighted centroid algorithm for transmitter localization in the presence of interference," IEEE Trans. Cognitive Commun. and Netw., vol. 2, no. 2, pp. 162-177, Jun. 2016.

[17] M. Abramowitz and I. A. Stegun, Handbook of Mathematical Functions with Formulas, Graphs, and Mathematical Tables. Dover, 1972.

[18] M. Haenggi, "On distances in uniformly random networks," IEEE Trans. Inf. Theory, vol. 51, no. 10, pp. 3584-3586, Oct. 2005.

[19] A. Prudnikov, Y. Brychkov, and O. Marichev, Integrals and Series, Volume 1: Elementary Functions. Gordon and Breach, 1986.

[20] J. Møller and R. P. Waagepetersen, Statistical Inference and Simulation for Spatial Point Processes. Chapman \& Hall/CRC, 2004.

[21] A. DasGupta, Probability for Statistics and Machine Learning. Springer-Verlag, 2011. 